\title{
Current uses and knowledge of medicinal plants in the Autonomous Community of Madrid (Spain): a descriptive cross-sectional study
}

Marta Sánchez ${ }^{1}$, Elena González-Burgos ${ }^{1}$, Irene Iglesias ${ }^{1}$, Rafael Lozano ${ }^{2}$ and María Pilar Gómez-Serranillos ${ }^{1 *}$

\begin{abstract}
Background: The usage of medicinal plants as a key component of complementary and alternative medicine, has acquired renewed interest in developed countries. The current situation of medicinal plants in Spain is very limited. This paper provides new insights and greater knowledge about current trends and consumption patterns of medicinal plants in the Autonomous Community of Madrid (Spain) for health benefits.

Methods: A descriptive cross-sectional study was designed for a population-based survey on medicinal plants. The data were collected (May 2018 to May 2019) using semi-structured face-to-face interviews in independent pharmacies, hospital centers and primary care health centers in the Autonomous Community of Madrid. The survey had 18 multiple choice and open-ended questions. Quantitative indices were calculated: Fidelity Level (FL), Use Value (UV) and Informants Consensus Factor (ICF). Chi-square test was used for data analysis.

Results: Five hundred forty-three people were interviewed. The majority of the participants (89.6\%) have used medicinal plants to treat health disorders in the past 12 months, mainly for digestive problems, sleep disorders and central nervous system diseases. A total of 78 plants were recorded, being Matricaria recutita, Valeriana officinalis, Tilia spp. and Aloe vera the most used. The highest UV was found for Mentha pulegium (UV 0.130) followed by Aloe vera (UV 0.097) and Vaccinium macrocarpon. (UV 0.080). The highest FL values were for Eucalyptus spp. (FL 90.47\%) for respiratory conditions and, Matricaria recutita (85.55\%) and Mentha pulegium (84.09\%) for digestive problems. The highest ICF corresponded to metabolism and depression (ICF =1), pain $(I C F=0.97)$, insomnia $(I C F=0.96)$ and anxiety (ICF $=0.95)$. Participants mostly acquired herbal medicines from pharmacies, herbal shops and supermarkets. Some side effects (tachycardia, dizziness and gastrointestinal symptoms) and potential interactions medicinal plantsdrugs ( $V$. officinalis and benzodiazepines) were reported.

Conclusion: Many inhabitants of the Autonomous Community of Madrid currently use herbal products to treat minor health problems. The most common consumer pattern are young women between 18 and 44 years of age with higher education. In order to confirm the pattern, further research should be focused to investigate current uses of medicinal plants in other Spanish regions.
\end{abstract}

Keywords: Medicinal plants, Autonomous Community of Madrid (CAM), Spain, Consumption patterns

\footnotetext{
* Correspondence: pserra@ucm.es

'Department of Pharmacology, Pharmacognosy and Botany, Faculty of Pharmacy, Universidad Complutense de Madrid (UCM), Madrid, Spain

Full list of author information is available at the end of the article
}

C C The Author(s). 2020 Open Access This article is licensed under a Creative Commons Attribution 4.0 International License, which permits use, sharing, adaptation, distribution and reproduction in any medium or format, as long as you give appropriate credit to the original author(s) and the source, provide a link to the Creative Commons licence, and indicate if changes were made. The images or other third party material in this article are included in the article's Creative Commons. licence, unless indicated otherwise in a credit line to the material. If material is not included in the article's Creative Commons licence and your intended use is not permitted by statutory regulation or exceeds the permitted use, you will need to obtain permission directly from the copyright holder. To view a copy of this licence, visit http://creativecommons.org/licenses/by/4.0/ The Creative Commons Public Domain Dedication waiver (http://creativecommons.org/publicdomain/zero/1.0/) applies to the data made available in this article, unless otherwise stated in a credit line to the data. 


\section{Background}

Complementary and alternative medicines (CAMs) represent different resources that complement or replace conventional therapies [1]. The World Health Organization's (WHO) strategy, 2014-2023, aims to strengthen the role of traditional medicine, emphasizing the importance of promoting and including the utilization of medicinal plants in the health systems of its member countries [2].

The use of medicinal plants has acquired a renewed interest in developed countries and constitutes the first therapeutic strategy for $80 \%$ of developing countries. The majority of the global population $(87.5 \%)$ uses traditional herbal medicine to treat health difficulties $[3,4]$. Moreover, the growing interest in the employ of medicinal plants is evidenced by the increase of systematic reviews and prevalence surveys about herbal medicines in the last 15 years [5]. In Europe and throughout the Mediterranean area, both wild-collected and purchased from herbalists, supermarkets and pharmacies, is reemerging. This renewed interest in traditional herbal medicine in more developed societies must be seen in the context of changes in the lifestyle, in which it enhances the concept of real and natural products. This leads consumers to perceive herbal medicine as a softer option for health issues [5-7].

Previous studies on medicinal plants in Spain are alternatively based on their traditional use [6,7]. All these preceding works aim to study the relationships between plants and human beings in the present and in the past, based on the understanding of herbal remedies which were traditionally used to treat disorders in different health situations [8]. However, the available information on current perspectives and uses of medicinal plants in Spain is very limited compared to other European countries and USA $[9,10]$ and additionally very restrictive to specific areas $[11,12]$.

On the other hand, there exists a widespread belief among population that herbal products, being from natural origin, are not harmful to health [13]. However, medicinal plants can interact with other drugs and thus cause adverse reactions $[13,14]$. The complete monographs of the German Commission E: Therapeutic Guide to Herbal Medicines includes more than 100 plants historically employed for their therapeutic properties but they are no longer recommended, since scientific evidence has shown potential toxicity or inefficiency [15].

Therefore, based on the state of the art, the aim of this study is to comprehend and deepen the current uses (consumption patterns, perceptions and attitudes) of medicinal plants in different regions of the Autonomous Community of Madrid (Spain), identifying the risks and precautions associated with its use and/or concomitant with conventional drugs.

\section{Methods}

\section{Study area}

The Autonomous Community of Madrid is the most densely populated territory in Spain (676 inhabitants per $\mathrm{km}^{2}$ ), it hosts the capital of Spain (Madrid). Most of the population is concentrated in Madrid Capital City and in its surrounding metropolitan areas. Even rural areas have Madrid as their referent in the urban lifestyle. The Autonomous Community of Madrid has a very diverse population in terms of its origin (being most of it from other Autonomous Communities), its cultural and socioeconomic terms [16]. This study has tried to represent different random localities with different social environments. In order to determine if the sample surveyed was representative of the population, the latest statistical data available on the website of Institute of Social Sciences (http://www.madrid.org/iestadis/) related to sex, age and occupation were analyzed.

\section{Study setting}

A descriptive cross-sectional study was designed for a population-based survey on medicinal plants. This research (PR016/04) was approved on November 2016 by the Ethics and Animal Experimentation Committee, Faculty of Pharmacy, University Complutense of Madrid (Spain).

\section{Questionnaire}

The questionnaire (Additional file 1), developed in Spanish language and designed for this study, was based on previous works on medicinal plants $[9,17,18]$ and reviewed by experts in traditional plant-based medicines and pharmacognosy and agreed with experts in public health in order to evaluate the structure, relevancy and clarity of the questions. Before gathering research data, a pilot study was conducted on a sample of 50 people to validate the degree of acceptance and understanding of the questionnaire. Minor modifications, based on the pilot survey, were made in the questionnaire. The final version of the questionnaire consisted on five differentiated parts with a total of 18 multiple choice and openended questions to achieve a better understanding of the knowledge and use of medicinal plants for healthseeking behavior. The first part with five questions collected information on demographic data, including age, gender, educational level, area of residence and occupation. The second part, with four issues, focused on the utilization of herbs for medicinal or health purposes (disease categories, frequency, therapeutic uses, types of medicinal plants - excluding multi-herbal drug combinations - and forms of administration). This part of the questionnaire included a definition of medicinal plants: "Plants that contain properties or compounds that can be used for therapeutic purposes or those that synthetize 
metabolites to produce useful drugs" [19] and, being respondents allowed freely to comment which medicinal plants they use to prevent or treat pathologies (open list of medicinal plants). Moreover, regarding the frequency of consumption, it has been considered frequent when the interviewee consumes medicinal plants at least once a month. The third part had three questions about where the consumer acquired the medicinal plants and information on their therapeutic uses. The fourth and the fifth sections containing both 3 questions, were related to the knowledge of potential side effects and identification of concomitant consumption of medicinal plants with conventional medicines, respectively.

\section{Data collection and sample size}

Data were collected on a Tablet computer by a research group from May 2018 to May 2019 using a face-to-face interview technique. Participants were recruited directly in a total of 30 independent pharmacies, hospitals and primary care health centers of different districts of the Capital City of Madrid and municipalities of the Autonomous Community of Madrid. The average number of interviewees from each place was from 15 to 20 .
Sample population interviewed was voluntary, randomly selected and previously informed (Fig. 1). Over the period of data collection, we conducted a total of 543 surveys. This sample size, based on population size, provides a margin error of $4 \%$ at $95 \%$ confidence level [20-22].

\section{Quantitative indices}

The quantitative indices Fidelity Level (FL), Use Value (UV) and Informants Consensus Factor (ICF) were calculated.

\section{Fidelity Level (FL)}

FL corresponds to the percentage of informants that use a certain medicinal plant to treat a specific condition and it is calculated as FL $(\%)=(\mathrm{Np} / \mathrm{N}) \times 100(\mathrm{~Np}$ : number of informants citing a certain medicinal plant to treat a specific condition and $\mathrm{N}$ : number of informants citing a medicinal plant to treat any given disease) [23]. This index is used to identify the most frequently used plants to treat a disease or condition.

\section{Use Value of species (UV)}

UV measures the relative importance of a medicinal plant to the informants and it is calculated as $\mathrm{UV}=$

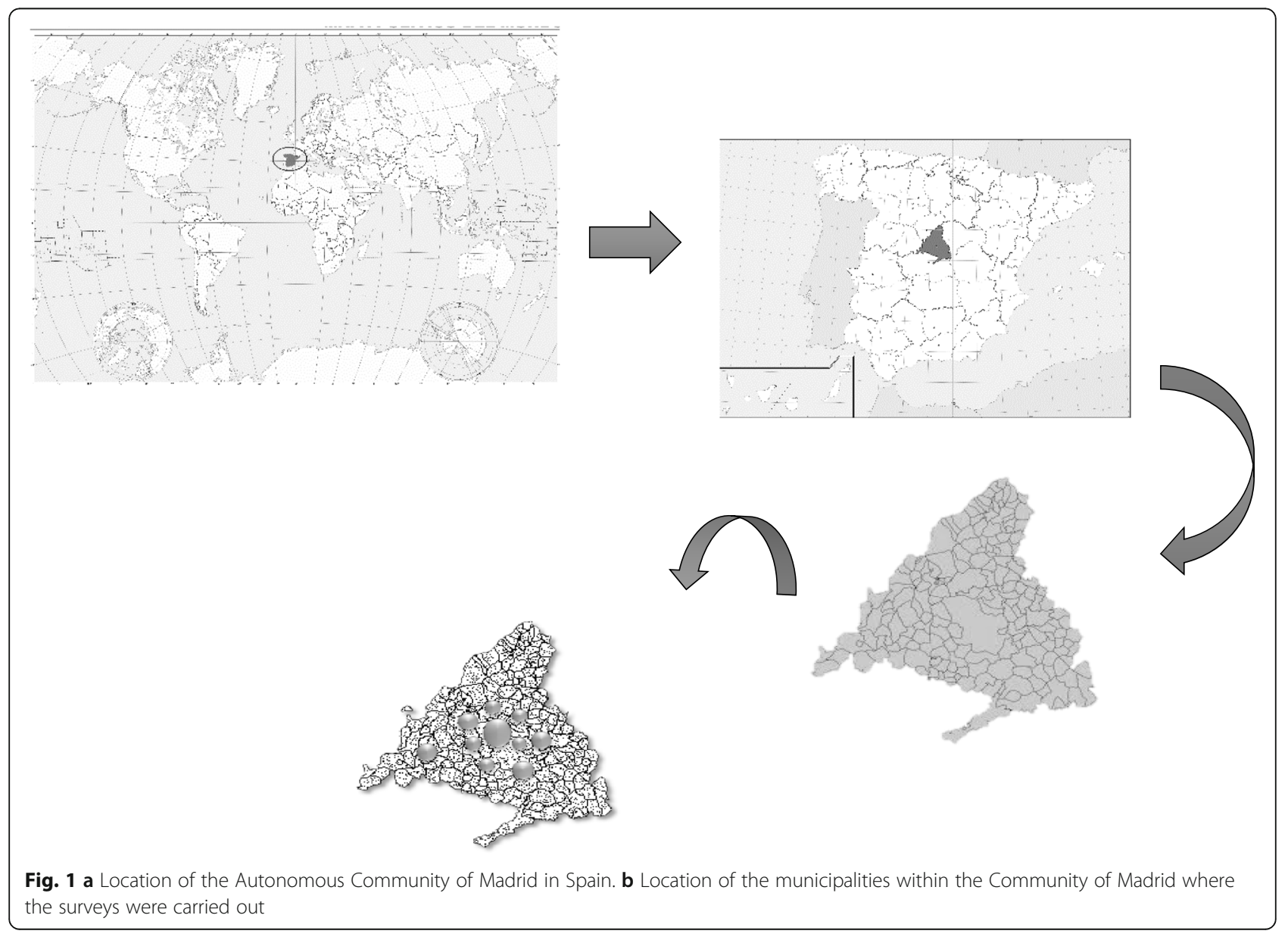


$\mathrm{Ui} / \mathrm{N}$ (Ui: number of citations for each medicinal plant and $\mathrm{N}$ : total number of informants). It is a quantitative parameter that indicates the relative importance of the different plant species in a community. It is useful to determine plants with the greatest use (most frequently used) in the treatment of a condition. It also allows knowing the confidence in the use and pharmacological characteristics of related plants $[17,24]$.

\section{Informants Consensus Factor (ICF)}

ICF estimates the user variability of medicinal plants and it is calculated as $\left(\mathrm{N}_{\mathrm{ur}}-\mathrm{N}_{\mathrm{t}}\right) /\left(\mathrm{N}_{\mathrm{ur}}-1\right)\left(\mathrm{N}_{\mathrm{ur}}\right.$ : number of used citations in each ailment category, and $\mathrm{Nt}$ : number of medicinal plants reported in each ailment category). This index is used to indicate to what extent the information is homogenous. The ranges obtained for this factor vary between 0 and 1 . A value close to 1 indicates a relatively high use of the medicinal plant, while a low value close to 0 shows that this plant species is not used by informants for the treatment of an ICF condition. This factor was originally developed by Trotter and Logan (1986) [25] and then readapted by Heinrich et al. $1998,2000[26,27]$.

\section{Data analysis}

All data were entered and stored in an Excel Spreadsheet. Frequencies and percentages were calculated using Microsoft Excel. Statistical analysis was performed using chi-square tests in Sigmaplot version 14.0, to analyze data with correlations between the frequency of medicinal plants and certain demographic characteristics. The level of statistical significance was $p<0.05$.

\section{Results}

\section{Demographic information}

Socio-demographic characteristics are shown in Table 1. Most participants were women $(n=382$; $70.3 \%)$. The most frequent age from the respondents was $18-44$ years $(n=340 ; 62.6 \%)$, followed by $45-64$ years old $(n=151 ; 27.8 \%)$ and finally by those over 65 years $(n=52 ; 9.6 \%)$. Regarding the level of education, the majority of interviewees had higher education $(n=417 ; 76.8 \%)$ while $3.2 \%$ of participants had basic education or vocational training (i.e. auto repair, plumbing). In reference to the occupation, more than half of participants were employees $(n=293 ; 54 \%)$, followed by students $(n=167 ; 30.8 \%)$, pensioners $(n=56$; $10.3 \%)$ and unemployed $(n=15 ; 2.8 \%)$.

\section{Uses and consumption patterns of medicinal plants}

The majority of the population interviewed $(n=491$, $89.6 \%$ ) used specifically medicinal plants to treat a disease or a health disorder, from which 20.1\% $(n=110)$ were habitual (more than 4 times/month) consumers
Table 1 Demographic characteristics of participation sample

\begin{tabular}{ll}
\hline Population characteristics & Answers $(\boldsymbol{N}=\mathbf{5 4 3}) \mathbf{n}(\%)$ \\
\hline Gender & $161(29.7 \%)$ \\
Male & $382(70.3 \%)$ \\
Female & \\
Age (years) & $340(62.6 \%)$ \\
$18-44$ & $151(27.8 \%)$ \\
$45-64$ & $52(9.6 \%)$ \\
$\geq 65$ & \\
Educational level & $56(10.3 \%)$ \\
Basic education & $70(12.9 \%)$ \\
Vocational training & $417(76.8 \%)$ \\
Higher education & \\
Occupation & $167(30.8 \%)$ \\
Student & $293(54 \%)$ \\
Employee & $15(2.8 \%)$ \\
Unemployed & $56(10.3 \%)$ \\
Pensioner & $12(2.2 \%)$ \\
Does not answer &
\end{tabular}

and $69.5 \%(n=381)$ were occasional users $(1-4$ times/ month). Only $10.4 \%$ of respondents $(n=57)$ had never consumed medicinal plants in the last 12 months (Table 2).

A total of 78 medicinal plants used for health problems, were identified in this study (Table 3). The average consumption was 2.3 medicinal plants by participant. The ten most commonly used medicinal plants were Matricaria recutita L. (24.8\%), Valeriana officinalis L. (20.5\%), Tilia spp. (13.6\%), Aloe vera L. (9\%), Camellia sinensis (L.) Kuntze (7.1\%), Mentha pulegium L. (6.9\%), Eucalyptus spp. (5.8\%), Passiflora incarnata L. (5.2\%), Rosa eglanteria L. (4.8\%) and Vaccinium macrocarpon Ait. (3.7\%) (Fig. 2). Some of these plants were also consumed in combined preparations, such as Valeriana officinalis, Passiflora incarnata and Eschscholzia californica Cham; however, these mixtures have not been taken into account in the study.

The uses of the medicinal plants were grouped into 12 categories. The most common therapeutic use (Table 4a)

Table 2 Frequency of use of herbal products (referring to the last 12 months) with therapeutically purposes among participation sample, according to range of age

\begin{tabular}{llll}
\hline $\begin{array}{l}\text { Frequency of use } \\
\text { of herbal products }\end{array}$ & Age & & \\
\cline { 2 - 4 } & $\mathbf{1 8 - 4 4} \mathbf{n}(\%)$ & $\mathbf{4 5 - 6 4} \mathbf{n}(\%)$ & $\mathbf{6 5} \mathbf{n ~ ( \% )}$ \\
\hline Frequently $^{\mathrm{a}}$ & $48(8.8 \%)$ & $39(7.1 \%)$ & $23(4.2 \%)$ \\
Occasionally $^{\mathrm{b}}$ & $256(46.7 \%)$ & $102(18.6 \%)$ & $23(4.2 \%)$ \\
Never & $35(6.4 \%)$ & $13(2.4 \%)$ & $9(1.6 \%)$ \\
\hline
\end{tabular}

a $>4$ times/month

${ }^{b_{1}}-4$ times/month 
Table 3 List of reported medicinal plants used (botanical name, family, disease/problem, mode of use, FL and UV)

\begin{tabular}{|c|c|c|c|c|c|c|}
\hline Medicinal plant & Family & Common name & Disease/problem & Mode of use & $\mathrm{FL}(\%)$ & uv \\
\hline Aesculus hippocastanum L. & Hipocastanaceae & Horse chestnut & Vascular problems & Extract/Capsule & 66.66 & 0.0055 \\
\hline Ajuga chamaepitys (L.) Schreb. & Lamiaceace & Bugle & Digestive problems & Infusions & 100 & 0.0018 \\
\hline Aloe vera (L.) Burm. $f$. & Asparagaceae. & Aloe vera & Calm pain, healing & Cream/gel & 55.55 & 0.097 \\
\hline Aloysia triphylla (L'Hér.) Britton & Verbenaceae & Herb louisa & $\begin{array}{l}\text { Digestive problems / Calm } \\
\text { pain }\end{array}$ & Infusion / capsule & 75 & 0.022 \\
\hline Althaea officinalis L. & Malvaceae & Marshmallow & Respiratory problems & Infusion/Capsule & 50 & 0.0036 \\
\hline Arctium lappa $\mathrm{L}$. & Compositae & Burdock & Digestive problems & Capsule & 100 & 0.0018 \\
\hline Argania spinosa (L.) Skeels & Sapotaceae & Argan & Healing & Essential oil & 66.66 & 0.0055 \\
\hline Arnica montana $\mathrm{L}$. & Compositae & Arnica & Calm pain & Cream & 66.66 & 0.016 \\
\hline $\begin{array}{l}\text { Aspalathus linearis (Burm. f.) R. } \\
\text { Dahlgren }\end{array}$ & Leguminosae & Rooibos & Digestive problems & Infusion & 100 & 0.0055 \\
\hline Calendula officinalis $\mathrm{L}$. & Compositae & Calendula & Calm pain & Cream & 12.5 & 0.014 \\
\hline Camellia sinensis (L.) Kuntze. & Theaceae & Tea plant & Digestive problems & Capsule/Infusion & 64.58 & 0.072 \\
\hline Cassia angustifolia Vahl. & Leguminosae & Senna & Digestive problems & Capsule & 33.33 & 0.016 \\
\hline Centella asiatica L. Urb. & Apiaceae & Asiatic pennywort & Healing & Cream & 33.33 & 0.0055 \\
\hline Cinnamomum verum J. Presl. & Lauraceae & Cinnamon & $\begin{array}{c}\text { Control sugar, } \\
\text { respiratory problems }\end{array}$ & Infusion & 33.33 & 0.0055 \\
\hline Citrus sinensis (L.) Osbeck & Rutaceae & Sweet Orange & Sleep disorders & Infusion & 66.66 & 0.055 \\
\hline Coffea arabica $\mathrm{L}$. & Rubiaceae & Arabica Coffe & Others (stimulant) & Infusion & 75 & 0.0073 \\
\hline Crataegus monogyna Jacq. & Rosaceae & Hawthorn & $\begin{array}{l}\text { Anxiety/nervousness } \\
\text { states }\end{array}$ & Capsule & 42.85 & 0.012 \\
\hline Cuminum cyminum $\mathrm{L}$. & Apiaceae & Cumin & Digestive problems & Infusion & 100 & 0.0018 \\
\hline Curcuma longa L. & Zingiberaceae & Turmeric & $\begin{array}{l}\text { Digestive problems, } \\
\text { calm pain }\end{array}$ & Capsule & 71.42 & 0.012 \\
\hline Cynara scolymus L. & Compositae & Globe artichoke & Digestive problems & Extract/Capsule & 88.88 & 0.016 \\
\hline Echinacea purpurea (L.) Moench & Compositae & Echinacea & Respiratory problems & Infusion & 46.15 & 0.023 \\
\hline $\begin{array}{l}\text { Eleutherococcus senticosus (Rupr. \& } \\
\text { Maxim.) Maxim. }\end{array}$ & Araliaceae & Siberian ginseng & $\begin{array}{l}\text { Others (stimulant, } \\
\text { anti-stress) }\end{array}$ & Extract/Capsule & 100 & 0.00368 \\
\hline Equisetum arvense $\mathrm{L}$. & Equisetaceae & Field horsetail & Digestive problems & Infusion/Extract & 68.96 & 0.033 \\
\hline Eschscholzia californica Cham. & Papaveraceae & California poppy & Sleep disorders & Capsule & 40 & 0.0092 \\
\hline Eucalyptus globulus Labill. & Myrtaceae & Eucalyptus & Respiratory problems & $\begin{array}{c}\text { Capsule/vapors/ } \\
\text { ointment }\end{array}$ & 90.47 & 0.071 \\
\hline Euphrasia rostkoviana Hayne & Orobanchaceae & Eyebright & Digestive problems & Infusion & 100 & 0.0018 \\
\hline Foeniculum vulgare Mill. & Apiaceae & Fennel & Digestive problems & Infusion & 100 & 0.020 \\
\hline Fucus vesiculosus $\mathrm{L}$. & Fucaceae & Bladder wrack & Others (laxative) & Capsule/Extract & 66.66 & 0.0055 \\
\hline Garcinia cambogia (Gaertn.) Desr. & Clusiaceae & Garcinia cambogia & Others (weight loss) & Capsule & 66.66 & 0.0055 \\
\hline Ginkgo biloba L. & Ginkgoaceae & Ginkgo & Vascular problems & Capsule & 57.14 & 0.012 \\
\hline Glycine $\max (\mathrm{L}$.$) Merr.$ & Leguminosae. & Soyabean & Others (menopause) & Capsule & 0 & 0.0073 \\
\hline Glycyrrhiza glabra L. & Leguminosae. & Licorice & Digestive problems & Capsule & 72.72 & 0.020 \\
\hline Grindelia robusta Nutt. & Compositae & Gumweed herb. & Respiratory problems & Syrups & 100 & 0.0018 \\
\hline Hamamelis virginiana $\mathrm{L}$. & Hamamelidaceae & Witch Hazel & Vascular problems & Capsule & 50 & 0.0036 \\
\hline $\begin{array}{l}\text { Harpagophytum procumbens (Burch.) } \\
\text { DC. ex Meisn. }\end{array}$ & Pedaliaceae & Devil's Claw & Calm pain & Capsules & 85.71 & 0.012 \\
\hline Hibiscus sabdariffa L. & Malvaceae & Roselle & Respiratory problems & Infusions & 100 & 0.0036 \\
\hline Hypericum perforatum $\mathrm{L}$. & Hyperidaceae & St. John's wort. & Depression & Capsules & 40 & 0.0092 \\
\hline Jasminum grandiflorum L. & Oleaceae & Jasmin & $\begin{array}{l}\text { Anxiety/nervousness } \\
\text { states }\end{array}$ & Infusions & 100 & 0.0018 \\
\hline Laurus nobilis L. & Lauraceae & Bay laurel & Digestive problems & Infusions & 50 & 0.0036 \\
\hline Lavandula angustifolia Mill. & Lamiaceae & Lavander & Sleep disorders & Infusions & 50 & 0.014 \\
\hline Malva sylvestris L. & Malvaceae & Common mallow & Respiratory problems & Infusions & 30 & 0.018 \\
\hline Matricaria recutita $\mathrm{L}$. & Compositae & German chamomille & Digestive problems & Infusions & 85.55 & 0.044 \\
\hline Melaleuca linariifolia Maiden \& Betche & Myrtaceae & Tea tree & Skin disorders & Essential oil & 50 & 0.0036 \\
\hline Melissa officinalis L. & Lamiaceae & Lemon balm & Sleep disorders & Infusions & 64.70 & 0.038 \\
\hline Mentha pulegium L. & Lamiaceae & Pennyroyal & Digestive problems & Infusions & 84.09 & 0.13 \\
\hline Mentha $\times$ piperita L. & Lamiaceae & Peppermint & Digestive problems & Infusions & 72.41 & 0.035 \\
\hline Monascus spp (Yeast rice) & Monascaceae & Red yeast rice & Others (cholesterol) & Capsules & 100 & 0.0073 \\
\hline Oenothera biennis $\mathrm{L}$. & Onagraceae & Evening primrose & $\begin{array}{c}\text { Others } \\
\text { (premenstrual syndrome) }\end{array}$ & Capsules & 50 & 0.011 \\
\hline Origanum vulgare L. & Lamiaceae & Oregano & Digestive problems & Infusions & 50 & 0.014 \\
\hline Panax ginseng C.A.Mey. & Araliaceae & Asiatic Ginseng & $\begin{array}{l}\text { Others (stimulant, anti- } \\
\text { stress) }\end{array}$ & Capsules & 100 & 0.0092 \\
\hline Passiflora incarnata $\mathrm{L}$. & Passifloraceae & Purple passionflower & Sleep disorders & Capsules/extract & 83.33 & 0.054 \\
\hline Paullinia cupana Kunth & Sapindaceae & Guarana & Others (stimulant) & Capsules & 100 & 0.0018 \\
\hline Peumus boldus Molina & Momiaceae & Boldo & Digestive problems & Infusions / capsules & 87.5 & 0.014 \\
\hline Pimpinella anisum $\mathrm{L}$. & Apiaceae & Aniseed & Digestive problems & Infusions & 85 & 0.032 \\
\hline Plantago major L. & Plantaginaceae & Broadleaf plantain & Respiratory problems & Infusions & 50 & 0.0036 \\
\hline Plantago ovata Phil. & Plantaginaceae & Spogel plantain & Digestive problems & $\begin{array}{c}\text { Capsules/oral } \\
\text { suspension powder }\end{array}$ & 85.71 & 0.012 \\
\hline Rhamnus purshiana DC & Rhamnaceae & Cascara buckthorn & Digestive problems & Extract/ capsules & 100 & 0.0092 \\
\hline Rhamnus frangula L. & Rhamnaceae & Alder buckthorn & Digestive problems & infusions/capsules & 100 & 0.0018 \\
\hline Rheum palmatum L. & Polygonaceae & Chinese rhubarb & Digestive problems & Capsules/infusions & 100 & 0.0018 \\
\hline Rosa eglanteria L. & Rosaceae & Sweet briar & Healing & Essential oil & 83.87 & 0.055 \\
\hline Rosa micrantha Borrer & Rosaceae & Smallflower sweetbrier & Others & Capsules & 100 & 0.0018 \\
\hline Rosmarinus officinalis $\mathrm{L}$. & Lamiaceae & Rosemary & Calm pain & Essential oil & 27.27 & 0.038 \\
\hline Ruscus aculeatus $\mathrm{L}$. & Asparagaceae & Butcher's-broom & Vascular problems & Capsules & 62.5 & 0.014 \\
\hline Serenoa repens (W. Bartram) Small. & Arecaceae & Saw palmetto & $\begin{array}{l}\text { Others (benign prostatic } \\
\text { hyperplasia) }\end{array}$ & Capsules & 33.33 & 0.0055 \\
\hline Silybum marianum (L.) Gaertn. & Compositae & Variegated thistle & Digestive problems & Extract/capsules & 87.5 & 0.014 \\
\hline $\begin{array}{l}\text { Simmondsia chinensis (Link) C.K. } \\
\text { Schneid }\end{array}$ & Simmondsiaceae & Jojoba & Healing & Essential oil & 100 & 0.0036 \\
\hline $\begin{array}{l}\text { Syzygium aromaticum (L.) Merr. \& } \\
\text { L.M. Perry }\end{array}$ & Myrtaceae & Clove & Digestive problems & Essential oil & 100 & 0.0018 \\
\hline $\begin{array}{l}\text { Taraxacum officinale (L.) Weber ex } \\
\text { F.H. Wigg. }\end{array}$ & Compositae & Dandelion & Digestive problems & $\begin{array}{l}\text { Infusions / } \\
\text { capsules }\end{array}$ & 100 & 0.0036 \\
\hline Thymus vulgaris $\mathrm{L}$. & Lamiaceae & Thyme & Respiratory problems & Infusions & 60.86 & 0.035 \\
\hline Tilia cordata Mill. & Malvaceae & Lime & Anxiety/nervousness states & Infusions & 70.83 & 0.052 \\
\hline Tribulus terrestris L. & Zygophyllaceae & Bendy-eye & $\begin{array}{l}\text { Hormone enhancer/ } \\
\text { Others (weight loss) }\end{array}$ & Capsules & 100 & 0.0018 \\
\hline Turnera diffusa Willd. ex Schult. & Passifloraceae. & Damiana & $\begin{array}{l}\text { Tonic/digestive } \\
\text { problems }\end{array}$ & Capsules & 100 & 0.0018 \\
\hline Urtica dioica $\mathrm{L}$. & Urticaceae & Stinging nettle & Calm pain & Capsules & 16.66 & 0.011 \\
\hline Vaccinium macrocarpon Aiton & Ericaceae & American cranberry & $\begin{array}{l}\text { Genitourinary } \\
\text { problems }\end{array}$ & Capsules & 76 & 0.08 \\
\hline Valeriana officinalis L. & Caprifoliaceae & Common Valerian & $\begin{array}{l}\text { Anxiety/nervousness } \\
\text { states/sleep disorders }\end{array}$ & Capsules & 76.38 & 0.034 \\
\hline Verbascum thapsus L. & Schrophulariaceae & Mullein & Respiratory problems & Infusions & 100 & 0.0018 \\
\hline Vitis vinifera $\mathrm{L}$. & Vitaceae & Grapevine & Vascular problems & Capsules & 80 & 0.0092 \\
\hline Zingiber officinale Rosc. & Zingiberaceae & Ginger & Digestive problems & Infusions & 54.54 & 0.02 \\
\hline
\end{tabular}




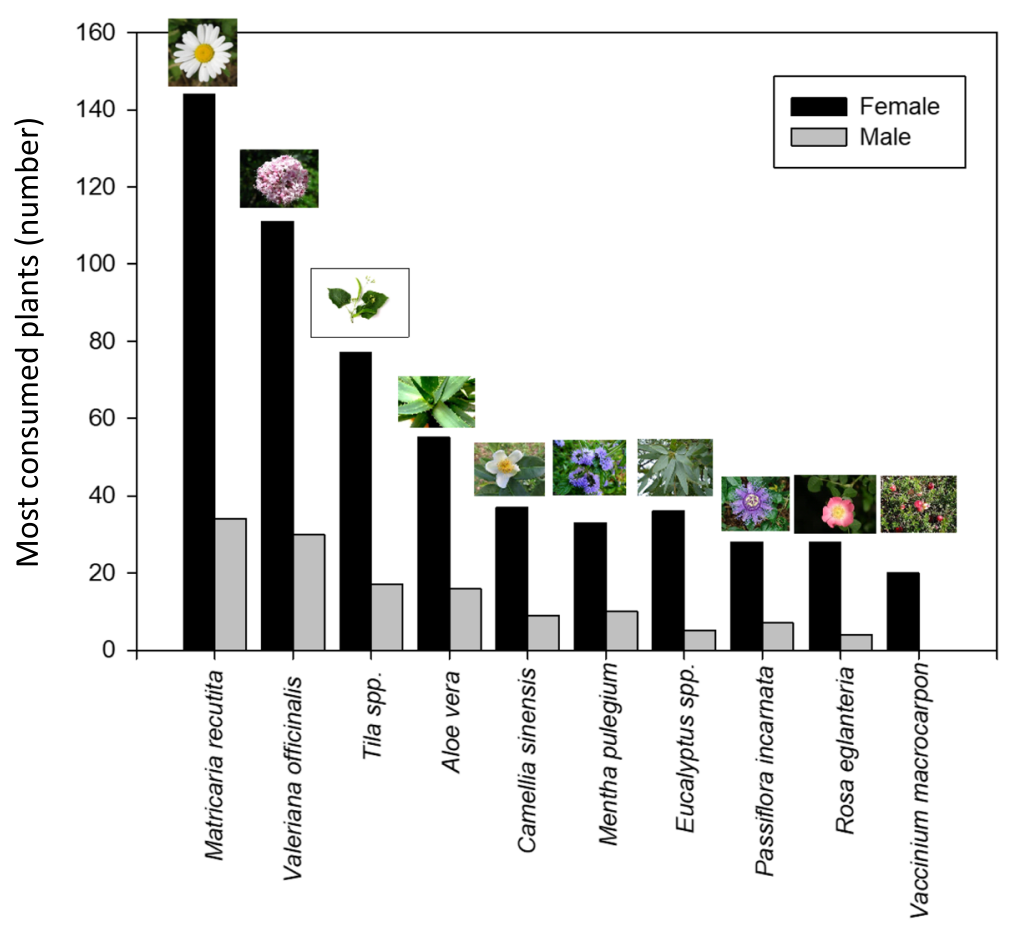

Fig. 2 The ten most consumed medicinal plants by the population of the Autonomous Community of Madrid in Spain according to the gender ( $\mathrm{n}$ total $=543 ; \mathrm{n}$ female $=382$ and $\mathrm{n}$ male $=161$ )

was for digestive problems such as intestinal gas and stomach cramps $(n=252,69 \%)$, followed by sleep disorders $(n=211,57.8 \%)$, anxiety and nervousness states $(n=166,45.5 \%)$ and, respiratory problems such as bronchitis and common cold ( $n=93,25.5 \%)$. Other less common therapeutically uses were genitourinary problems $(n=42,11.5 \%)$, vascular problems $(n=31,8.5 \%)$, blood pressure control $(n=12,3.3 \%)$, blood sugar levels $(n=8$, $2.2 \%)$ and depression $(n=8,2.2 \%)$.

The most popular form of consumption was as herbal infusion $(n=369,75.8 \%)$, followed by tablets/capsules $(n=210,43.1 \%)$ and creams $(n=121,24.8 \%)$ (Table 4b).

\section{Quantitative indices}

\section{The Fidelity Level (FL)}

The results of the Fidelity Level for the 10 most cited medicinal plants showed that the highest values were for Eucalyptus spp. (FL 90.47\%) for respiratory conditions followed by Matricaria recutita (85.55\%) and Mentha pulegium (84.09\%) for digestive problems treatment and, Valeriana officinalis (76.38\%) for insomnia (Table 5).

\section{The Use Value (UV)}

UV calculations revealed that the highest value was found for Mentha pulegium (UV 0.130) followed by Aloe vera (UV 0.097) and Vaccinium macrocarpon. (UV 0.080). These were followed by Camellia sinensis (UV 0.072) and Eucalyptus spp. (UV 0.071) (Table 6).

\section{Informant Consensus Factor (ICF)}

The highest ICF value found corresponds to metabolism and depression $(\mathrm{ICF}=1)$ followed by pain $(\mathrm{ICF}=0.97)$, insomnia $(\mathrm{ICF}=0.96)$ and anxiety $(\mathrm{ICF}=0.95)($ Table 7$)$.

\section{Place of acquisition preferences and therapeutic resources}

Regarding to the place where herbal products were acquired, almost half of the participants preferred pharmacies $(n=253,51.9 \%)$ followed by herbal shops $(n=209$, $42.9 \%)$ and supermarkets $(n=170,34.9 \%)$, being. The internet resulted in the last position (2.7\%) (Table 8).

Most interviewers initiated the consumption of medicinal plants for prevention and treatment following the recommendations of friends and family $(n=226,46.4 \%)$, being less who started by their own initiative $(n=216$, 44.3\%) (Table 8). The information concerning the therapeutically uses of medicinal plants came mainly from family and friends $(n=234,48.1 \%)$, followed by pharmacist $(n=210,43.1 \%)$ and the internet $(n=160,32.8 \%)$ (Table 8).

\section{Subjective perception of risks and precautions of medicinal plants}

Half of the respondents $(n=227,46.6 \%)$ believed that medicinal plants could cause adverse reactions such as conventional drugs do while the other half of the sample population did not $(n=260,53.4 \%)$. Moreover, it was 
Table 4 (A) Main uses for medicinal plants among the survey sample population. (B) Herbal products most used for therapeutic purposes among the population interviewed. Several possible answers were possible for both questions

\begin{tabular}{|c|c|c|c|}
\hline & \multicolumn{3}{|l|}{ Age } \\
\hline & 18-44 n, (\%) & 45-64 n, (\%) & $\geq 65 \mathrm{n},(\%)$ \\
\hline \multicolumn{4}{|l|}{ A) } \\
\hline \multicolumn{4}{|l|}{ Clinical purposes } \\
\hline Anxiety / nervousness states & $118(32.3 \%)$ & $38(10.4 \%)$ & $10(2.7 \%)$ \\
\hline Blood pressure control & $4(1.1 \%)$ & $5(1.4 \%)$ & $3(0.8 \%)$ \\
\hline Calm the pain & $45(12.3 \%)$ & $20(5.5 \%)$ & $6(1.6 \%)$ \\
\hline Control sugar & $3(0.8 \%)$ & $2(0.5 \%)$ & $3(0.8 \%)$ \\
\hline Depression & $4(1.1 \%)$ & $2(0.5 \%)$ & $2(0.5 \%)$ \\
\hline Digestive problems & $141(38.6 \%)$ & $85(23.3 \%)$ & $26(7.1 \%)$ \\
\hline Genitourinary problems & $24(6.6 \%)$ & $14(3.8 \%)$ & $4(1.1 \%)$ \\
\hline Wound healing & $54(14.8 \%)$ & $16(4.4 \%)$ & $1(0.3 \%)$ \\
\hline Others & $54(14.8 \%)$ & $39(10.7 \%)$ & $10(2.7 \%)$ \\
\hline Respiratory problems & $55(15.1 \%)$ & $34(9.3 \%)$ & $4(1.1 \%)$ \\
\hline Sleep disorders & $132(36.2 \%)$ & $65(17.8 \%)$ & $14(3.8 \%)$ \\
\hline Vascular problems & $12(3.3 \%)$ & $17(4.7 \%)$ & $2(0.5 \%)$ \\
\hline \multicolumn{4}{|l|}{ B) } \\
\hline \multicolumn{2}{|c|}{ Forms of consumption of medicinal plants } & \multicolumn{2}{|l|}{ Answers $\mathrm{n},(\%)$} \\
\hline \multicolumn{2}{|c|}{ Creams (i.e. Aloe vera, Rosa eglanteria, Arnica montana) } & \multicolumn{2}{|l|}{$121(24.8 \%)$} \\
\hline \multicolumn{2}{|l|}{ Essential oils (i.e. Eucalyptus spp.) } & \multicolumn{2}{|l|}{$84(17.2 \%)$} \\
\hline \multicolumn{2}{|c|}{ Herbal teas (i.e. Equisetum arvense) } & \multicolumn{2}{|l|}{$369(75.8 \%)$} \\
\hline \multicolumn{2}{|c|}{ Syrups (i.e. Eleutherococcus senticosus) } & \multicolumn{2}{|l|}{$37(7.6 \%)$} \\
\hline \multicolumn{2}{|c|}{ Tablets, capsules (i.e. Valeriana officinalis, Vaccinium macrocarpon, Passiflora incarnata) } & \multicolumn{2}{|l|}{$210(43.1 \%)$} \\
\hline
\end{tabular}

investigated if any of the respondents had suffered any side effect when consuming herbal products for therapeutic purposes. Of those respondents, 17 (3.5\%) reported that they had suffered some adverse reaction such as anxiety, tachycardia, dizziness and gastrointestinal symptoms (Table 9).

The potential risk in respect of interactions between medicinal plants and conventional drugs was also investigated. Several respondents have consumed medicinal plants along with conventional medicines ( $n=103$; 21.1\%) (Table 10a). Generally, patients do not perceive the need to separate medicinal plants consumption from other drugs. Moreover, interviewees have acknowledged not have received information from health institutions about potential medicinal plants and conventional drugs interactions.

It is revealed that there are several different medicinal plants which were concomitantly consumed with

Table 5 Fidelity Level (FL) of the ten most consumed medicinal plants

\begin{tabular}{|c|c|c|c|}
\hline Medicinal Plants & Main therapeutic uses & No. of claimed uses reports & FL (\%) \\
\hline Valeriana officinalis $\mathrm{L}$. & Insomnia & 110 & 76.38 \\
\hline Aloe vera $L$. & Wound healing & 40 & 55.55 \\
\hline Matricaria recutita $\mathrm{L}$. & Digestive problems (i.e. flatulence, stomatitis, and gastrointestinal spasms) & 154 & 85.55 \\
\hline Tilia spp. & Anxiety & 68 & 70.83 \\
\hline Rosa eglanteria $L$. & Wound healing & 26 & 36.11 \\
\hline Camellia sinensis (L.) Kuntze & Asthenia & 27 & 49.09 \\
\hline Mentha pulegium L. & Digestive problems (i.e. flatulence, dyspepsia) & 37 & 84.09 \\
\hline Eucalyptus spp. & Common cold & 38 & 90.47 \\
\hline Passiflora incarnata L. & Anxiety & 22 & 59.45 \\
\hline Vaccinium macrocarpon Ait. & Cystitis & 19 & 76 \\
\hline
\end{tabular}


Table 6 Use Value (UV) of the ten most consumed medicinal plants

\begin{tabular}{|c|c|c|c|c|c|}
\hline Plant specie & Common name & Part(s) used & Methods of use & Reported uses (per claimed respondents) & UV \\
\hline Valeriana officinalis $\mathrm{L}$. & Valerian & Root & Oral, infusion & $\begin{array}{l}\text { Anxiety / nervousness states, blood pressure control, } \\
\text { gastrointestinal disorder, sleep disorders }\end{array}$ & 0.034 \\
\hline Aloe vera $\mathrm{L}$. & Aloe vera & Gel & Topical & $\begin{array}{l}\text { Anxiety / nervousness states, calm pain, gastrointestinal } \\
\text { disorders, sleep disorders, vascular problems, wound healing, }\end{array}$ & 0.097 \\
\hline Matricaria recutita $\mathrm{L}$. & Chamomile & Flower & Infusion & $\begin{array}{l}\text { Anxiety / nervousness states, blood pressure control, calm } \\
\text { pain, gastrointestinal disorders, genitourinary problems, sleep } \\
\text { disorders, wound healing. }\end{array}$ & 0.044 \\
\hline Tilia spp, & Tila & Leaves & Infusion & $\begin{array}{l}\text { Anxiety / nervousness states, blood pressure control, calm } \\
\text { pain, gastrointestinal disorders, sleep disorders. }\end{array}$ & 0.052 \\
\hline Rosa eglanteria $\mathrm{L}$. & Rose Hip & Oil & Topical & $\begin{array}{l}\text { Anxiety / nervousness states, sleep disorders, vascular } \\
\text { problems wound healing. }\end{array}$ & 0.055 \\
\hline Camellia sinensis (L.) Kuntze & Thea & Leaves & Infusion & $\begin{array}{l}\text { Anxiety / nervousness states, gastrointestinal disorders, } \\
\text { sleep disorders, }\end{array}$ & 0.072 \\
\hline Mentha pulegium L. & Pennyroyal & Summit & Infusion & $\begin{array}{l}\text { Anxiety / nervousness states, blood pressure control, calm } \\
\text { pain, gastrointestinal disorders, sleep disorders. }\end{array}$ & 0.13 \\
\hline Eucalyptus spp. & Eucalyptus & Leaves & Topical & $\begin{array}{l}\text { Anxiety / nervousness states, gastrointestinal disorders, } \\
\text { respiratory disorders, }\end{array}$ & 0.071 \\
\hline Passiflora incarnata L. & Passiflora & Aerial part & Infusion & Anxiety / nervousness states, sleep disorders & 0.054 \\
\hline Vaccinium macrocarpon Ait & Red blueberry & Fruit & Oral & Calm pain, genitourinary problems & 0.08 \\
\hline
\end{tabular}

conventional drugs (ibuprofen, levonorgestrel/ethinylestradiol, paracetamol and omeprazole) (Table 10b). It is concluded that Matricaria recutita and Valeriana officinalis were the medicinal plants most commonly consumed together with conventional drugs.

The percentage of patients who did not inform doctors or pharmacists of medicinal plants consumption while using other medicines was 65.3\% (Table 10a).

\section{Discussion}

This work reveals new insights and greater knowledge about the main reasons and current consumption mode of medicinal plants in the population of the Autonomous Community of Madrid for health benefits.

The Community of Madrid has a very varied population and it is very densely populated. Therefore, data from our study were compared with those available from the Institute of Social Sciences to find out whether the surveyed population is representative of the population of this Spanish region. As evidenced demographic parameters are representative (i.e. active population percentage which is $43.6 \%$ and range of age which are $55.3 \%$ for $18-44,27.3 \%$ for $45-64$ and $17.3 \%$ for $\geq 65$ years) [16].

Regarding medicinal plants, it was unconcluded that it was higher than the one estimated for other Spanish cities [12]. The main reasons for this finding are the consumer's perception of efficacy and safety as well as the easy access. In this study, the most common consumption pattern of medicinal plants is young women, between 18 and 44 years of age, with higher education. There is statistically significant differences in

Table 7 Informant Consensus Factor (ICF) per medicinal plant category

\begin{tabular}{llll}
\hline Ailment category & Number of claimed medicinal plants & Number of claimed citations & ICF \\
\hline Anxiety / nervousness states & 9 & 166 & 12 \\
Blood pressure control & 3 & 71 & 0.95 \\
Calm the pain & 3 & 8 \\
Control Sugar & 1 & 8 \\
Depression & 1 & 252 \\
Gastrointestinal disorder & 32 & 42 \\
Genitourinary problems & 5 & 93 \\
Respiratory disorders & 17 & 211 \\
Sleep disorders & 8 & 31 \\
Vascular problems & 5 & 71 \\
Wound healing & 5 & 0.97 \\
\hline
\end{tabular}


Table 8 People who have recommended the use of medicinal plants, place of acquisition and sources of information among the general population surveyed. Several possible answers were possible for both questions

\begin{tabular}{|c|c|c|}
\hline Questions & Possible responses & n (\%) \\
\hline \multirow[t]{4}{*}{ Who recommends the medicinal plants you use? } & Doctor recommendation & $67(13.7)$ \\
\hline & Own initiative & $216(44.3)$ \\
\hline & Pharmacists advice & $162(33.3)$ \\
\hline & Recommended by friends / family / acquaintances & $226(46.4)$ \\
\hline \multirow[t]{5}{*}{ Where do you acquire mainly medicinal plants? } & Supermarkets & $170(34.9)$ \\
\hline & Herbal shops & $209(42.9)$ \\
\hline & Internet & $13(2.7)$ \\
\hline & Others (i.e. street market) & $36(7.4)$ \\
\hline & Pharmacy & $253(51.9)$ \\
\hline \multirow[t]{5}{*}{ Where do you mainly get information about the uses of medicinal plants? } & Doctor & $71(14.6)$ \\
\hline & Family and friends & $234(48.1)$ \\
\hline & Internet & $160(32.8)$ \\
\hline & Other means of communication (magazines, TV ...) & $82(16.8)$ \\
\hline & Pharmacist & $210(43.1)$ \\
\hline
\end{tabular}

consumption frequency related to gender respondents, being higher in women $(P<0,001)$. This high prevalence in the preference of medicinal plants by the female gender has been also confirmed in previous studies [28]. As surveys have been conducted in different health centers, the fact that participants were predominantly women may be due that visits to pharmacies, nurses and doctors in Spain are more frequent in women [29] alongside satisfaction with complementary and alternative medicines [30]. Moreover, a statistically significant finding related to age ranges was found [respondents aged 18-44 consumed medicinal plants more often than those in 45-64 age range $(P=0,010)$ and even more often than those $\geq 65$ years $(P<0,001)]$. This pattern, contrasts with studies performed in other parts of Europe where the frequency of consumption is higher in older people rather than in younger people [31]. Moreover, studies from the USA found that medicinal plants consumption is more frequent in middle-aged people [10]. These differences may lie in the area where study was conducted, economic level and consumer trends. Particularly, the Autonomous Community of Madrid has the highest Gross Domestic Product per capita in Spain. In addition, it is one of the Spanish regions most influenced by urbanization and where there is not such a strong connection to traditional use of medicinal plants as in other areas of Spain. Furthermore, there is a growing trend, especially amongst younger people with higher educational level, to use natural products to succeed a healthy lifestyle and mentality $[3,31]$.

Table 9 Survey responses related to side effects of medicinal plants among the general population surveyed

\begin{tabular}{lll}
\hline Questions & Possible responses & n (\%) \\
\hline Do you think that medicinal plants may cause side effects? & Yes & 227 (46.6) \\
& No & 260 (53.4) \\
Have you had any reaction or side effect when consuming medicinal plants? & Yes & (3.5) \\
& No & W6.5) \\
If the previous answer is YES: & reaction? & \\
Chamomile / vomiting & & \\
Dandelion / dizziness & & \\
Ginseng / nervous, diarrhea and tachycardia & \\
Guarana / tachycardia Sen / diarrhea and tachycardia & \\
St. John's wort/ interaction similar to the shock & \\
Tea / anxiety, palpitations & \\
Valerian / sleepiness the next day &
\end{tabular}


Table 10 (A) Survey responses related to concomitant consumption of medicinal plants and conventional drugs. (B) Main conventional drugs and medicinal plants that are consumed concomitantly ( $n=$ number of there associations have been reported in the survey)

\begin{tabular}{|c|c|c|c|c|}
\hline \multicolumn{5}{|l|}{ A) } \\
\hline & \multicolumn{2}{|r|}{ Questions } & Possible responses & $\mathrm{n}(\%)$ \\
\hline & \multirow{3}{*}{\multicolumn{2}{|c|}{ Do you usually consume medicines and medicinal plants concomitantly? }} & Yes & $103(21.1)$ \\
\hline & & & No & $297(61.0)$ \\
\hline & & & Sometimes & $87(17.9)$ \\
\hline & \multirow{2}{*}{\multicolumn{2}{|c|}{ Do you communicate to your doctor / pharmacist that you consume conventional drugs and medicinal plants concomitantly? }} & Yes & $66(34.7)$ \\
\hline & & & No & $124(65.3)$ \\
\hline \multicolumn{5}{|l|}{ B) } \\
\hline \multirow{2}{*}{\multicolumn{2}{|c|}{$\begin{array}{l}\text { Medicinal plant } \\
\text { Aloe vera }\end{array}$}} & \multicolumn{3}{|l|}{ Conventional drugs } \\
\hline Aloe ver & & \multicolumn{3}{|l|}{ Ibuprofen $(n=3)$, levonorgestrel/ethinylestradiol $(n=1)$, metronidazole $(n=1)$, paracetamol $(n=2)$, tamoxifen $(n=1)$} \\
\hline \multicolumn{2}{|c|}{ Articum lappa } & \multicolumn{3}{|l|}{ Ibuprofen $(n=1)$} \\
\hline \multirow{2}{*}{\multicolumn{2}{|c|}{$\begin{array}{l}\text { Camellia sinensis } \\
\text { Cassia angustifolia }\end{array}$}} & \multicolumn{3}{|c|}{ Acetylsalicylic acid $(n=1)$, amoxicillin $(n=2)$, atenolol $(n=1)$, iron $(n=1)$, ibuprofen $(n=5)$, loratadine $(n=1)$, naproxen sodium $(n=1)$, omeprazole $(n=2)$, paracetamol $(n=3)$} \\
\hline & & \multicolumn{3}{|l|}{ Aripiprazole $(\mathrm{n}=1)$, quetiapine $(\mathrm{n}=1)$} \\
\hline \multicolumn{2}{|c|}{ Centella asiatica } & \multicolumn{3}{|l|}{ Ebastine $(n=1)$, ibuprofen $(n=1)$} \\
\hline \multicolumn{2}{|c|}{ Cynara scolymus } & \multicolumn{3}{|l|}{ Diosmin $(n=1)$, omeprazole $(n=1)$, salbutamol $(n=1)$, tiotropium bromure $(n=1)$} \\
\hline \multicolumn{2}{|c|}{ Echinacea purpurea } & \multicolumn{3}{|l|}{ Alprazolam (n=1), clomipramine $(n=1)$, ibuprofen $(n=1)$, metamizol $(n=1)$, paracetamol $(n=1)$} \\
\hline \multicolumn{2}{|c|}{ Equisetum arvense } & \multicolumn{3}{|c|}{ Atorvastatin $(n=1)$, clorazepate $(n=1)$, diazepam $(n=1)$, ibuprofen $(n=2)$, levonorgestrel/ethinylestradiol $(n=1)$, lorazepam, $(n=1)$, valsartan/hydrochlorothiazide $(n=1)$} \\
\hline \multicolumn{2}{|c|}{ Eschscholtzia californica } & \multicolumn{3}{|c|}{ Iron (n=1), salmeterol/fluticasone propionate $(n=1)$} \\
\hline Eucalypt & & Atorvastatin $(n=1)$, ibuprofen $(n=2)$, paracetamol $(n=3)$, valsartan/hydrochlorothiazide $(n=1)$ & & \\
\hline Foenicul & vulgare & Levonorgestrel/ethinylestradiol $(n=1)$, omeprazole $(n=1)$, tryptizol $(n=1)$ & & \\
\hline Ginkgo b & & Ibuprofen $(n=1)$ & & \\
\hline Laurus n & & Ibuprofen $(n=1)$ & & \\
\hline Malva sy & & Levonorgestrel/ethinylestradiol $(n=2)$ & & \\
\hline Matricar & ecutita & $\begin{array}{l}\text { Almagate }(n=1) \text {, atenolol }(n=1) \text {, atorvastatin }(n=1) \text {, dutasteride/tamsulosin }(n=1) \text {, enalapril }(n=2) \text {, irbesartan }(n=1) \text {, iron }(n) \text {, } \\
\text { mesalazine }(n=1) \text {, naproxen sodium }(n=1) \text {, omeprazole }(n=1) \text {, paracetamol }(n=14) \text {, pravastatin }(n=1) \text {, simethicone }(n=1) \text {, } \\
\text { valsartan/hydrochlorothiazide }(n=1)\end{array}$ & $\begin{array}{l}\text { vonorgestrel/ethinylestr } \\
\text { tatin }(\mathrm{n}=1) \text {, sitagliptin/m }\end{array}$ & $\begin{array}{l}=2) \text {, lormetazepam }(n=1), \\
n(n=1) \text {, tiotropium }(n=1)\end{array}$ \\
\hline Melissa & inalis & Alprazolam $(n=1)$, paracetamol $(n=3)$, ibuprofen $(n=2)$ & & \\
\hline Mentha & gium & Almagate ( $n=1)$, ibersartan/hydrochlorothiazide ( $n=1)$, ibuprofen $(n=6)$, levonorgestrel/ethinylestradiol $(n=2)$, paracetam & , rosuvastatine $(n=1)$ & \\
\hline Panaxgi & & Amoxicillin $(n=1)$, ibuprofen $(n=1)$ & & \\
\hline Passiflor & carnata & Clorazepate $(n=1)$, budesonide/formoterol $(n=1)$, ibuprofen $(n=3)$, iron $(n=1)$, salmeterol/fluticasone propionate $(n=1)$ & & \\
\hline Paullinia & & Amoxicillin (n=1), ibuprofen ( $n=1)$ & & \\
\hline Peumus & & Atorvastatin $(n=1)$, hydrochlorothiazide $(n=1)$, valsartan $(n=1)$ & & \\
\hline Pimpine & nisum & Levonorgestrel/ethinylestradiol $(n=2)$ & & \\
\hline Rhamnu & ingula & Ethinylestradiol/drospirenone $(\mathrm{n}=1)$ & & \\
\hline Rhamnu & rshiana & Aripiprazole $(n=1)$, ethinylestradiol/drospirenone $(n=1)$, quetiapine $(n=1)$ & & \\
\hline Rosa egl & & Levonorgestrel/ethinylestradiol $(n=1)$ & & \\
\hline Ruscusa & & Chondroitin sulfate $(\mathrm{n}=1)$ & & \\
\hline Sabal se & & Paracetamol $(n=1)$ & & \\
\hline Tilia spp & & Acetylsalicylic acid ( $n=1)$, buproprion $(n=1)$, enalapril $(n=1)$, ibuprofen $(n=1)$, irbersartan $(n=1)$, paracetamol $(n=8)$, pravast & $=1$ ), sitagliptin/metformi & , topical corticosteroids $(\mathrm{n}=1)$ \\
\hline Urtica di & & Ibuprofen ( $n=1)$, paracetamol $(n=1)$ & & \\
\hline Vacciniu & Syrtillus & Losartan $(n=1)$ & & \\
\hline Vacciniu & xycoccus & Fosfomycin (n=2), ciprofloxacin $(n=2)$, levonorgestrel/ethinylestradiol $(n=2)$ & & \\
\hline Valerian & ficinalis & $\begin{array}{l}\text { Anastrozol }(n=1) \text {, atenolol }(n=1) \text {, doxilamine }(n=1) \text {, dutasteride/tamsulosin }(n=1) \text {, escitalopram }(n=1) \text {, heparin }(n=1) \text {, ibup } \\
\text { levotiroxine }(n=1) \text {, lorazepam }(n=1) \text {, lormetazepam }(n=1) \text {, tiotropium bromure }(n=1) \text {, omeprazole }(n=2) \text {, paracetamol }(n=) \\
\text { corticosteroid }(n=1) \text {, trazodone hydrochloride }(n=1) \text {, vitamin } D(n=1)\end{array}$ & $\begin{array}{l}n=3) \text {, iron }(n=1) \text {, levonor } \\
\text { tidine }(n=1) \text {, salmeterol })\end{array}$ & $\begin{array}{l}\text { ethinylestradiol }(n=1) \text {, } \\
\text { one propionate }(n=1) \text {, topical }\end{array}$ \\
\hline
\end{tabular}

One of the limitations found in former published studies on prevalence of medicinal plants consumption, unlikely to the one presented, is on the one hand that "medicinal plants" concept is not properly defined, and on the other hand, a list of medicinal plants is providing limiting the knowledge of their use [5]. Of the 78 identified plants, women reported using 72 while men reported 49. Moreover, most people surveyed use them appropriately in relation to diseases for which they are found to be effective. There were no significant differences $(p=0.242)$ in medicinal plants consumption between female and male. However, preferences for some medicinal plants were found among gender. Melissa officinalis L, Cynara scolymus L., Echinacea angustifolia DC, Equisetum arvense L. and Mentha piperita L. were preferred by women whereas Vitis vinifera L. and Tribulus terrestris L. were preferred by men. Moreover, in this study, Vaccinium macrocarpon Ait. Consumption was exclusive to women in order to prevent uncomplicated acute lower urinary tract infections recurrence.
Women's urethra is shorter than that of men's allowing bacteria rapid access to the urinary bladder [32].

It is necessary to emphasize that some of the medicinal plants consumed by the population of the Autonomous Community of Madrid are considered as threatened/vulnerable/endangered by the IUCN Red List. These plant species include in this Red list are Aesculus hippocastanum (vulnerable), Arnica montana (least concern), Coffea arabica (endangered), Ginkgo biloba (endangered), Laurus nobilis (least concern), Rhamnus purshiana (least concern) and Tilia cordata (least concern). Particularly, those plant species classified as least concern are not considered to be at threat from extinction and, the future conservation actions are aimed at controlling agriculture practices and include an international legislation. However, Aesculus hippocastanum is classified as vulnerable because this plant species suffer from severe defoliation by the invasive insect pest Cameraria ohridella. The conservation actions consists on Cameraria ohridella control and research, ex situ 
cultivation and to reduce human impacts. On the other hand, Coffea arabica and Ginkgo biloba are endangered plant species. The main threats to Coffea arabica are pests (i.e. Hypothenemus hampei), diseases (i.e. Coffee Berry Disease), deforestation (mainly in Ethiopia) and climate change (i.e. high temperatures). There are several conservation actions for Coffea arabica such as exsitu conservation and, education and awareness programs. Finally, Ginkgo biloba is threatened because its logging and wood harvesting. The conservation action for this specie has been widespread in cultivation. It is therefore important that investigations with these species follow the guidelines "IUCN Policy Statement on Research Involving Species at Risk of Extinction" that guarantee the increase and survival of these plant species, bearing in mind that the conservation of these research sources is of clear scientific interest, and in the case of our study, of great therapeutic interest [33-39].

Regarding forms of consumption, the effectiveness of medicinal plants depends on the correct use and preparation. Decoction and infusion are the main preparation methods for herbal teas of roots, barks and seeds. Herbal teas are closely linked to self-medication, being this form of administration not suitable for active principles with narrow therapeutic margin. Tablets/capsules are commonly used for medicinal plants oral administration because of good bioavailability, therapeutic adherence and patient comfort [40].

Concerning accessibility to medicinal plants, most of the herbs are freely available in different places for its acquisition, even at supermarkets (i.e. Matricaria recutita, Camellia sinensis and Mentha pulegium) whereas there are other medicinal plants that are only available in local pharmacies and herbal shops (i.e. Verbascum thapsus and Ajuga chamaepitys). Participants' perception is that medicinal plants dispensed in pharmacies have better quality and efficiency than those from other acquisition places; however, medicinal plants bought in pharmacies are more expensive than in other sales establishments. This explains why the purchase of medicinal plants in supermarkets and herbal shops is very high. This pattern of herbal products acquisition for therapeutic purposes has also been observed in other countries [41]. However, within Spain, patients from a social security primary health care center in Barcelona bought medicinal plants first in herbal shops, then in supermarket and in pharmacies in third place [12]. The role of the pharmacist is consolidated as the health professional and expert in medicinal plants and pharmacy offices as a reference in the dispensation of medicinal plants, offering quality guarantees.

Due to the wide traditional utilization of medicinal plants and the limited existing clinical trials, there is a lack of scientific evidence on the efficacy and safety of medicinal plants [4]. Adverse drug reactions is defined as "all noxious and unintended responses to a medicinal product" [42, 43]. There is a common perception of safety of medicinal plants as "natural" and "harmless", which could lead to an under-reporting of adverse reactions. Adverse reactions may be due both to medicinal plants and to other factors (i.e. adulteration, lack of botanical identification) [44]. Studies conducted on natural products' perception for health, show an increase in the demand for information about medicinal plants $[45,46]$. It is necessary to include medicinal plants consumption in the usual medical history to identify possible adverse reactions and drug interactions [47]. Many health professionals have not received academic preparation on medicinal plants during their Degree studies [48]. In Spain, only pharmacists receive university education on medicinal plants. This lack of knowledge is a limiting factor when health professionals recommend medicinal plants and identify possible adverse reactions and interactions. The need to include medicinal plants in undergraduate training to the rest of health professionals is presumed.

Currently, there are a paucity of robust data on interactions between medicinal plants and conventional medicines [49]. However, it has been found that certain plants can lead to therapeutic inefficiency or drug toxicity. There is evidence of interactions for Hyperycum perforatum L. with digoxin, indinavir and cyclosporines [50]. Moreover, Ginkgo biloba L. Mant. Pl. can increase insulin elimination or interfere with omeprazole [51]. Furthermore, and in relation to the medicinal plants, that are more consumed concomitantly in this study, there are evidences of pharmacodynamics interactions between $M$. recutita and lormetazepam, $M$. officinalis and alprazolam, and $V$. officinalis and lormetazepam, increasing hypnotic effect of these benzodiazepines [51]. The clinical effects of the interactions depend on patient (age, genetic and pathologies), medicinal plants (species, dose and duration) and concomitant medication (dose, activity and posology) making it difficult to detect interactions if health personnel do not know its use.

Finally, several participants told that neither they reported medicinal plants consumption to these health professionals nor did they ask. This leads to a potential underreporting of adverse reactions and interactions with medicinal plants and, supports the need in the academic training of health sciences personnel to include subjects of medicinal plants in undergraduate degree.

\section{Conclusions}

In this paper, we have explored medicinal plant uses, consumption patterns and attitude towards medicinal plants of the population of the Autonomous Community of Madrid that attend health-related centers. This study 
shows that although the Autonomous Community of Madrid is not a region of Spain with a long tradition in the use of medicinal plants, many inhabitants currently use herbal products (i.e. M. recutita, $V$. officinalis, Tilia, $A$. vera. and $C$. sinensis) to treat, mainly, minor health problems (i.e. digestive problems and sleep disorders). All the reported medicinal plants have been extensively used in different countries, not identifying neither new records nor new therapeutic activities. These medicinal plants are mainly acquired in pharmacies, herbal shops and supermarkets. The most common consumer pattern of medicinal plants are young women between 18 and 44 years of age with higher education. It has been proved that one of the main reasons for the use of medicinal plants is that the surveyed population has the perception that being natural means harmless.

Moreover, in the present work a correct use of medicinal plants-therapeutic benefits has been detected. However, the high percentage of self-medication may increase the problem of lack of adverse reaction registration and/or drug interactions. Medicinal plants consumption is a matter to consider in the control of pharmacological treatments of the patients. This will guarantee safety, efficacy and quality in the use of medicinal plants, thus constituting an integral health system. According to the results of the study, the need for studies and research to predict the future use of medicinal plants is verified to ensuring the best quality of traditional herbal remedy.

Furthermore, taking into account that studies on current uses of medicinal plants in Spain are very limited, it would be interesting in future research to approach other regions in Spain to have deeper knowledge of the current situation, using and adapting the tolls of this work.

\section{Supplementary information}

Supplementary information accompanies this paper at https://doi.org/10. 1186/s12906-020-03089-x.

Additional file 1. Survey on medicinal plants. The questionnaire was developed in Spanish language and designed for this study.

\section{Abbreviations \\ CAM: Complementary and alternative medicine; FL: Fidelity Level; UV: Use Value; ICF: Informants Consensus Factor}

\section{Acknowledgements}

We thank all the participants.

\section{Authors' contributions}

MS and EGB contributed to the study conception, investigation and writing the original draft. MPGS was responsible to the study conception and the supervision of the study as well as for the writing (review and editing). RL and II contributed to writing (review and editing). All authors read and approved the manuscript.
Funding

None.

\section{Availability of data and materials}

The data will be accessible by contacting the corresponding author of this study.

\section{Ethics approval and consent to participate}

This research (PR016/04) was approved on November 2016 by the Ethics and Animal Experimentation Committee, Faculty of Pharmacy, University

Complutense of Madrid (Spain). Informed consent was verbal according to Spanish legislation (Ley 41/2002).

\section{Consent for publication}

Not applicable.

\section{Competing interests}

The authors declare that they have no competing interests.

\section{Author details}

${ }^{1}$ Department of Pharmacology, Pharmacognosy and Botany, Faculty of Pharmacy, Universidad Complutense de Madrid (UCM), Madrid, Spain. ${ }^{2}$ Department of Chemistry in Pharmaceutical Sciences, Faculty of Pharmacy, UCM, Madrid, Spain.

Received: 6 February 2020 Accepted: 16 September 2020

Published online: 14 October 2020

\section{References}

1. Mainardi T, Kapoor S, Bielory L. Complementary and alternative medicine: herbs, phytochemicals and vitamins and their immunologic effects. J Allergy Clin Immunol. 2009;123(2):283-94.

2. World Health Organization. WHO traditional medicines strategy 2014-2023. Geneva: WHO; 2013.

3. Devesa F, Pellicer J, Ginestar F, Borghol A, Bustamante M, Ortuño J, Ferrando I, Llobera C, Sla A, Miñana M, Nolasco A, Fresquet JL. Consumo de hierbas medicinales en los pacientes de consultas externas de digestivo. Gastroenterol Hepatol. 2004;27(4):244-9.

4. Parveen A, Parveen B, Parveen R, Ahmad S. Challenges and guidelines for clinical trial of herbal drugs. J Pharm Bioallied Sci. 2015;7:329-33.

5. McLay JS, Pallivalappila AR, Shetty A, Pande B, Al Hail M, Stewart D. Asking the right Question'. A comparison of two approaches to gathering data on 'Herbals'. Use in Survey Based Studies PLoS One. 2016;11.

6. Alarcón R, Pardo de Santayana M, Priestley C, Morales R, Heinrich M. Medicinal and local food plants in the south of Alava (Basque Country, Spain). J Ethnopharmacol. 2015;176:207-24.

7. Calvo MI, Cavero RY. Medicinal plants used for ophthalmological problems in Navarra (Spain). J Ethnopharmacol. 2016;190:212-8.

8. Castillo García E, Martínez I. Manual de fitoterapia. Barcelona: ElservierMasson; 2016. p. 536. ISBN: 978-84-458-1797-1.

9. Knotek K, Verner V, Chaloupkova P, Kokoska L. Prevalence and use of herbal products in the Czech Republic: over-the-counter survey among adult pharmacies clients. Complement Ther Med. 2012;20:199-206. https://doi. org/10.1016/j.ctim.2011.12.010.

10. Craft R, McClure KC, Corbett S, Ferreira MP, Stiffarm AM, Kindscher K. Ethnic differences in medicinal plant use among University students: a crosssectional survey of self-reported medicinal plant use at two Midwest Universities. BMC Complement Altern Med. 2015;15:192.

11. Alonso MJ, Capdevila C. Estudio descriptivo de la dispensación de fitoterapia en la farmacia catalana. Revista Fitoterapia. 2005;5:31-9.

12. Baulies Romero G, Torres R, Martín A, Roig AM, Royo I, Orfila F. Hábitos de consumo de plantas medicinales en un centro de salud de Barcelona. Revista Fitoterapia. 2011;11:45-51.

13. Ekor $M$. The growing use of herbal medicines: issues relating to adverse reactions and challenges in monitoring safety. Front Pharmacol. 2014;4(7):1-10.

14. Shugaba Al, Umar MBT, Uzokwe C, Umaru GJ, Muhammad MB, Shinku F, Rabiu AM, Mathew R. The effect of Yoyo cleanser bitters on the cerebellum of adult male Wistar rat. Sky J Med Med Sci. 2014;2(5):21-30.

15. Blumenthal M, Goldberg A, Brinckmann J. Herbal medicine: expanded commission E monographs, Boston; 2000.

16. INEbase. National Statistics Institute on-line database. 2017. 
17. Mahomoodall MF, Ramalingum N. An investigation into the consumption patterns, attitude, and perception of Mauritians towards common medicinal food plants. J Herbal Med. 2015;5(2):99-112.

18. Delgoda R, Younger N, Barrett C, Braithwaite J, Davis D. The prevalence of herbs use in conjunction with conventional medicines in Jamaica. Compl Ther Med. 2010;18:13-20.

19. World Health Organization. WHO guidelines on good agricultural and collection practices for medicinal plants. Geneva: WHO; 2007.

20. Daniel J. Sampling essentials practical guidelines for making sampling choices. Washington DC: Sage Publications Inc; 2011.

21. Morales P. Tamaño necesario de la muestra: ¿Cuántos sujetos necesitamos? Madrid: Universidad Pontificia Comillas; 2012. Facultad de Humanidades.

22. García-García JA, Reding-Bernal A, López-Alvarenga JC. Cálculo del tamaño de la muestra en investigación en educación médica. Inv Ed Med. 2013;2(8): 217-24.

23. Friedman J, Yaniv Z, Dafni A, Palewitch D. A preliminary classification of the healing potential of medicinal plants, based on a rational analysis of an ethnopharmacological field survey among Bedouins in the Negev desert. Israel J Ethnopharmacol. 1986;16:275-87.

24. Cakilcioglu U, Khatun S, Turkoglu I, Hayta S. Ethnopharmacological survey of medicinal plants in Maden(Elazig-Turkey). J Ethnopharmacol. 2011:137:469-86.

25. Trotter R, Logan M. Informant consensus: a new approach foridentifying potentially effective medicinal plants. In: Etkin NL, editor. Plants in indigenous medicine and diet: biobehavioural approaches. Bedfort Hills: Redgrave Publishers; 1986. p. 91-112.

26. Heinrich M. Ethnobotany and its role in drug development. Phytother Res. 2000;14(7):479-88.

27. Heinrich M, Ankli A, Frei B, Weimann C, Sticher O. Medicinal plants in Mexico: healers' consensus and cultural importance. Soc Sci Med. 1998;47:1859-71.

28. Bardia A, Nisly NL, Zimmerman MB, Gryzlak BM, Wallace RB. Use of herbs among adults based on evidence-based indications: findings from the National Health Interview Survey. Mayo Clin Proc. 2007;82(5):561-6.

29. Ministerio de Sanidad, Consumo y Bienestar Social. Actividad y Calidad de los Servicios Sanitarios Informe Anual del Sistema Nacional de Salud. Ed. Secretaría General Técnica Centro de publicaciones Nipo en línea. 2017 731-19-046-0.

30. Zahn R, Perry N, Perry E, Mukaetova-Ladinska EB. Use of herbal medicines: Pilot survey of UK users' views. Complement Ther Med. 2019;44:83-90.

31. Raal A, Volmer D, Sõukand R, Hratkevitš S, Kalle R. Complementary treatment of the common cold and flu with medicinal plants--results from two samples of pharmacy customers in Estonia. PLoS One. 2013;8:1-6.

32. Harper M, Fowlis G. Management of urinary tract infections in men. Trends in urology. Gynaecol Sex Health. 2007;12:30-5.

33. Allen D J, Khela S. Aesculus hippocastanum (errata version published in 2018). The IUCN Red List of Threatened Species 2017. 2017; e. T202914A122961065

34. Falniowski A, Bazos I, Hodálová I, Lansdown R, Petrova A. Arnica montana. The IUCN Red List of Threatened Species 2011. 2011: e.T162327A5574104.

35. Moat J, O'Sullivan RJ, Gole T, Davis AP. Coffea arabica (amended version of 2018 assessment). The IUCN Red List of Threatened Species 2020. 2020: e. T18289789A174149937.

36. Sun W. Ginkgo biloba. The IUCN Red List of Threatened Species 1998. 1998: e.T32353A9700472.

37. Khela S, Wilson B. Laurus nobilis. The IUCN Red List of Threatened Species 2018. 2018: e.T203351A119996864.

38. Stritch L. Frangula purshiana. The IUCN Red List of Threatened Species 2018 2018: e.T61957071A61957074.

39. Rivers MC, Barstow M, Khela S. Tilia cordata. The IUCN Red List of Threatened Species 2017. 2017: e.T203360A68079373.

40. López Luengo MT. Formas de administración más habituales de plantas medicinales. Offarm. 2002;21:11-161.

41. Dragoeva AP, Koleva VP, Nnaova ZD, Koynova TV, Jordanova PK. A study on current status of herbal utilization in Bulgaria. Part 1: application of herbal medicines. Sci Res Essays. 2015;10:168-76.

42. World Health Organization. Adverse drug reactions monitoring. Geneva: WHO; 2008.

43. European Medicines Agency. Guidelines on good pharmacovigilance practices. 2019

44. Saw JT, Bahari MB, Ang HH, Lim YH. Potential drug-herb interaction with antiplatelet/anticoagulant drugs. Complementary Ther Clin Pract. 2006;12: 236-41.
45. Walji R, Boon H, Barnes J, Welsh S, Austin Z, Baker GR. Reporting natural health product related adverse drug reactions: is it the pharmacist's responsibility? Int J Pharm Pract. 2011;19(6):383-91.

46. Hirschkorn $\mathrm{K}$, Walji R, Boon $\mathrm{H}$. The role of natural health products (NHPs) in dietetic practice: results from a survey of Canadian dietitians. BMC Complement Alternat. 2013;13:156

47. Cordero JA. Uso racional de la fitoterapia: una asignatura pendiente. FMC. 1998:5:414.

48. Mattos G, Camargo A, Sousa CA, Zeni AL. Medicinal plants and herbal medicines in primary health care: the perception of the professionals. Salud Colect. 2018;23:3735-44.

49. Fischer FH, Lewith G, Witt CM, Linde K, von Ammon K, Cardini F, Falkenberg $T$, Fønnebø V, Johannessen H, Reiter B, Uehleke B, Weidenhammer W, Brinkhaus B. High prevalence but limited evidence in complementary and alternative medicine: guidelines for future research. BMC Complement Alternat. 2014;14:46.

50. Soleymani S, Bahramsoltani R, Rahimi R, Abdollahi M. Clinical risks of St John's Wort (Hypericum perforatum) co-administration. Expert Opin Drug Metab Toxicol. 2017;13:1047-62.

51. Hu Z, Yang X, Ho PC, Chan SY, Heng PW, Chan E, Duan W, Koh HL, Zhou S. Herb-drug interactions: a literature review. Drugs. 2005;65:1239-82.

\section{Publisher's Note}

Springer Nature remains neutral with regard to jurisdictional claims in published maps and institutional affiliations.
Ready to submit your research? Choose BMC and benefit from:

- fast, convenient online submission

- thorough peer review by experienced researchers in your field

- rapid publication on acceptance

- support for research data, including large and complex data types

- gold Open Access which fosters wider collaboration and increased citations

- maximum visibility for your research: over $100 \mathrm{M}$ website views per year

At BMC, research is always in progress.

Learn more biomedcentral.com/submissions 